\title{
Predicted Mean Residence Time Infinity
}

National Cancer Institute

\section{Source}

National Cancer Institute. Predicted Mean Residence Time Infinity. NCI Thesaurus. Code C85793.

The predicted mean residence time (MRT) extrapolated to infinity. 